\title{
COPD: algorithms and clinical management
}

\author{
Rosa Faner ${ }^{1,2}$ and Alvar Agustí ${ }^{1,2,3}$ \\ Affiliations: ${ }^{1}$ Centro Investigación Biomédica En Red Enfermedades Respiratorias (CIBERES), Spain. \\ ${ }^{2}$ Fundació Clínic per a la Recerca Biomèdica, Institut d'investigacions Biomèdiques August Pi i Sunyer \\ (IDIBAPS), Barcelona, Spain. ${ }^{3}$ Pulmonary Service, Respiratory Institute, Hospital Clinic, University of \\ Barcelona, Barcelona, Spain.
}

Correspondence: Rosa Faner, CELLEXP2A, c/Casanova 143, Barcelona 08036, Spain. E-mail: rfaneraclinic.cat

@ERSpublications

Can algorithms help the practicing clinician to prescribe the best treatment? http://ow.ly/44yF30fwCc9

Cite this article as: Faner R, Agustí A. COPD: algorithms and clinical management. Eur Respir J 2017; 50: 1701733 [https://doi.org/10.1183/13993003.01733-2017].

When you are invited to write an Editorial on a given paper, you are expected to provide a strong personal opinion that will both attract the readers' interest whilst giving food for thought. Here, we have been asked to try to achieve both goals with respect to the paper by Burgel et al. [1] on the use of a simple algorithm for the identification of clinical chronic obstructive pulmonary disease (COPD) phenotypes published in this same issue of the European Respiratory Journal (ERJ).

As any other scientific paper, this particular one has strengths and limitations. Among the former: 1) it addresses an important clinical topic, the heterogeneity of COPD; 2) it uses novel analytical methodologies and, most importantly, it validates main observations in a second, independent cohort; and 3) it suggests a simple way to stratify COPD patients in the clinic in relation to a specific outcome (all-cause mortality). Authors merit congratulations for these three achievements. Yet, and apologies for playing the devil's advocate role here as requested, it also has some limitations that deserve comment, including: 4) what is really new for practice, beyond common clinical sense that dictates that patients with severe airflow limitation and those aged with more comorbidities (cardiovascular diseases and diabetes) have worse outcomes, and 5) how can this algorithm help the practicing clinician (and, more importantly, the patient) to prescribe the best treatment possible? Let's discuss these five points in some detail below.

\section{COPD heterogeneity}

That COPD is heterogeneous means that not all its pathological manifestations are present in all patients at any given time point and/or at different time points in the same patient [2]. No doubt, this is an important topic with clear managerial and prognostic implications. To address it, the concept of "clinical phenotypes" was originally introduced to stratify COPD patients into groups of individuals with similar characteristics and outcomes that may therefore merit similar treatment [3, 4]. Unsupervised statistical methods, such as cluster analysis, can be used to identify clinical phenotypes in a data-driven, unbiased manner and, in fact, several studies have used them to identify key clinical features determining the heterogeneity of COPD [5-14]. Very recently, however, CASTALDI et al. [15] performed identical clustering analyses in 10 different cohorts in North America and Europe ( $\mathrm{n}=17146$ COPD patients) and found: 1) that the reproducibility of COPD clustering subtypes across these studies was modest; and 2) that COPD heterogeneity was better characterised by continuous disease traits, coexisting in varying degrees within the same individual, rather than by mutually exclusive COPD subtypes (or phenotypes). However, it is

Received: Aug 232017 | Accepted after revision: Aug 312017

Conflict of interest: Disclosures can be found alongside this article at erj.ersjournals.com

Copyright CERS 2017 
important to note that, at variance with the work of CASTALDI et al. [15], Burgel et al. [1] determined the reproducibility of their findings with respect to a specific (and important) outcome (all-cause mortality). This suggests, as highlighted by CASTALDI et al. [15], that "it may be useful to use differences in clinically relevant outcomes to...define...COPD subtypes". The implication would then be that "there may be multiple distinct sets of subtypes that depend on the specific clinical outcome of interest" [15].

\section{Methodology and validation}

Burgel et al. [1] made a significant contribution to the field by using classification and regression trees to develop a machine learning-based algorithm that uses fixed thresholds for clinical variables and is able to allocate individual COPD patients to five categories related to 3-year all-cause mortality. The method was developed using the baseline characteristics of 2409 patients from the French/Belgian COPD cohorts [5] sorted into five classification categories predefined by unbiased clustering. Then, the algorithm was applied to 3651 patients of the COPD Cohorts Collaborative International Assessment (3CIA) initiative [16] proving the reproducibility of results in relation to 3 year mortality. In concordance with previous clustering results, the two groups with higher risk of mortality were older patients with cardiovascular comorbidity and diabetes, and younger patients with more severe respiratory disease $[5,13]$.

\section{Clinical implications}

Burgel et al. [1] showed that it is feasible to use a set of relatively simple clinical characteristics (age, body mass index, forced expiratory volume in $1 \mathrm{~s}$ (\% predicted), modified Medical Research Council dyspnoea scale, number of exacerbations in the previous 12 months, and presence/absence of cardiovascular comorbidities and/or diabetes) to objectively stratify patients into groups of high and low mortality risk. If implemented into the clinical setting, this stratified medicine approach might be useful to define the follow-up strategy for groups of patients in order to optimise healthcare delivery and, hopefully, reduce mortality.

\section{What is really new?}

As our grandmothers would likely say, do you really need all this complex analytical approach to know that patients with more severe airflow limitation and those with more concomitant diseases have poor prognosis? Any experienced clinician already knew it. So, in our opinion, the real value of this paper is the proof of concept that algorithms can be developed with simple clinical variables and that they can be useful for COPD stratification and risk assessment. Thus, this work reinforces the now well-accepted idea that not all COPD patients are equal and that, therefore, appropriate stratification and, eventually, individualisation of treatment [17] (see the following section) is of paramount importance.

\section{Individualisation of treatment}

So, how do we put all this together? We believe that, at the end of the day, what really matters in practice is not to know to what group (subtype, cluster or phenotype, however you prefer to call them) the patient that you have in front of you in your office belongs but what is the best treatment that you can (should) offer to this unique individual. From this perspective, we think that the large cross-cohort analysis by CASTALDI et al. [15] discussed above is important, since it shows that COPD heterogeneity (if not related to a specific outcome) is better described by continuous disease traits, coexisting in varying degrees in the same patient, rather than by mutually exclusive COPD subtypes. Hence, accepting the risk of being biased, we would therefore suggest that in clinical practice, when confronted with a single patient, the concept of "treatable traits" (as discussed elsewhere in the ERJ [18]) is the way forward to individualise treatment, while algorithms like that developed by BURGEL et al. [1] can provide complementary information on the individual-patient risk in relation to specific outcomes. Treatable traits can be recognised phenotypically (observable characteristics of an organism) or through validated biomarkers that inform on the presence of specific mechanisms of disease (or endotypes) [18]. Importantly, at variance with the "phenotype" approach, they can coexist in the same individual, and change with time or as a result of treatment [18]. They can be pulmonary, extrapulmonary, and/or social, behavioural and environmental [18]. Needless to say that, as recently agreed in a European Respiratory Society research seminar on this topic, this strategy needs formal validation [19].

In any case, as requested, we have given you our personal opinion on this interesting paper by BURGEL et al. [1] and we hope that we might have also provided you with some food for thought. Now, it is up to you to agree or disagree. Have a nice day.

\section{References}

1 Burgel P-R, Paillasseur J-L, Janssens W, et al. A simple algorithm for the identification of clinical COPD phenotypes. Eur Respir J 2017; 50: 1701034. 
2 Agusti A, Gea J, Faner R. Biomarkers, the control panel and personalized COPD medicine. Respirology 2016; 21: 24-33.

3 Friedlander AL, Lynch D, Dyar LA, et al. Phenotypes of chronic obstructive pulmonary disease. COPD 2007; 4: 355-384.

4 Han MK, Agusti A, Calverley PM, et al. Chronic obstructive pulmonary disease phenotypes: the future of COPD. Am J Respir Crit Care Med 2010; 182: 598-604.

5 Burgel P-R, Paillasseur J-L, Caillaud D, et al. Clinical COPD phenotypes: a novel approach using principal component and cluster analyses. Eur Respir J 2010; 36: 531-539.

6 Pistolesi M, Camiciottoli G, Paoletti M, et al. Identification of a predominant COPD phenotype in clinical practice. Respir Med 2008; 102: 367-376.

7 Cho MH, Washko GR, Hoffmann TJ, et al. Cluster analysis in severe emphysema subjects using phenotype and genotype data: an exploratory investigation. Respir Res 2010; 11: 30.

8 Garcia-Aymerich J, Gómez FP, Benet M, et al. Identification and prospective validation of clinically relevant chronic obstructive pulmonary disease (COPD) subtypes. Thorax 2011; 66: 430-437.

9 Rennard SI, Locantore N, Delafont B, et al. Identification of five chronic obstructive pulmonary disease subgroups with different prognoses in the ECLIPSE cohort using cluster analysis. Ann Am Thorac Soc 2015; 12: 303-312.

10 Castaldi PJ, Dy J, Ross J, et al. Cluster analysis in the COPDGene study identifies subtypes of smokers with distinct patterns of airway disease and emphysema. Thorax 2014; 69: 415-422.

11 Pinto LM, Alghamdi M, Benedetti A, et al. Derivation and validation of clinical phenotypes for COPD: a systematic review. Respir Res 2015; 16: 50.

12 Burgel PR, Paillasseur JL, Roche N. Identification of clinical phenotypes using cluster analyses in COPD patients with multiple comorbidities. Biomed Res Int 2014; 2014: 420134.

13 Burgel PR, Roche N, Paillasseur JL, et al. Clinical COPD phenotypes identified by cluster analysis: validation with mortality. Eur Respir J 2012; 40: 495-496.

14 Burgel PR, Paillasseur JL, Peene B, et al. Two distinct chronic obstructive pulmonary disease (COPD) phenotypes are associated with high risk of mortality. PLoS One 2012; 7: e51048.

15 Castaldi PJ, Benet M, Petersen H, et al. Do COPD subtypes really exist? COPD heterogeneity and clustering in 10 independent cohorts. Thorax 2017; 72: 998-1006.

16 Soriano JB, Lamprecht B, Ramírez AS, et al. Mortality prediction in chronic obstructive pulmonary disease comparing the GOLD 2007 and 2011 staging systems: a pooled analysis of individual patient data. Lancet Respir Med 2015; 3: 443-450.

17 Vogelmeier CF, Criner GJ, Martinez FJ, et al. Global Strategy for the Diagnosis, Management, and Prevention of Chronic Obstructive Lung Disease 2017 Report: GOLD Executive Summary. Eur Respir J 2017; 49: 1700214.

18 Agusti A, Bel E, Thomas M, et al. Treatable traits: toward precision medicine of chronic airway diseases. Eur Respir J 2016; 47: 410-419.

19 Agusti A, Bafadhel M, Beasley R, et al. Precision medicine of airway diseases: moving to clinical practice. Eur Respir J 2017; 50: 1701655. 